\section{Optimal Paint Gun Orientation in Spray Paint Applications-Experimental Results}

\author{
Pål Johan From, Member, IEEE, Johan Gunnar, and \\ Jan Tommy Gravdahl, Senior Member, IEEE
}

\begin{abstract}
In this paper, we present the experimental results of a new spray paint algorithm presented in previous publications. Both theory and simulations indicate that the proposed method allows a robotic manipulator to paint a given surface using substantially lower joint torques than with conventional approaches. In this paper, we confirm this by implementing the algorithm on an ABB robot and we find that the joint torques needed to follow the trajectory are substantially lower than for the conventional approach.

The approach presented is based on the observation that a small error in the orientation of the end effector does not affect the quality of the paint job. It is far more important to maintain constant velocity for the entire trajectory. We thus propose to allow a small error in the specification of the end-effector orientation, and we show how this allows us to obtain a higher constant speed throughout the trajectory. In addition, to improve the uniformity of the paint coating we are also able perform the paint job in less time.
\end{abstract}

Note to Practitioners-This paper presents several experiments that verify previously obtained theoretical results. For a large class of tasks where the robot is to hold a pointing device, such as a painting gun or a heating device, we show through experiments on a real robot, that we can increase the speed at which the robot tool traverses the surface. In spray painting it is for example far more important to keep a constant speed than to hold the paint gun orthogonal to the surface. The method proposed is to implement a slightly different planning algorithm in turns by allowing a small orientation error. The trajectory planner will then use the freedom obtained by allowing this orientation error to follow the trajectory with a higher constant velocity. The experiments presented in this paper show that we are able to reduce the energy needed to paint a surface with about $50 \%$ or increase the speed at which the paint gun traverses the surface with more than $50 \%$.

Index Terms-Spray painting, assembly-line manufacturing, modeling, empirical studies.

\section{INTRODUCTION}

$\mathbf{I}$ N ROBOTICS research, empirical studies are extremely important in order to validate algorithms and simulation results. Even though simulation tools are becoming increasingly accurate, they can never compare to real-world experiments. In this paper, we present the empirical data obtained by implementing three different spray paint algorithms and running these on a robot manipulator in our lab. The algorithms compute a trajectory in joint space for which the end-effector follows a predefined path. The joint torques are then measured for the different approaches and compared to the conventional approach. The

Manuscript received May 07, 2010; revised September 17, 2010; accepted October 06, 2010. Date of publication November 15, 2010; date of current version April 06, 2011. This paper was recommended for publication by Associate Editor Y.-B. Jia and Editor S. Sarma upon evaluation of the reviewers' comments. This work was supported in part by the Norwegian Research Council and the TAIL IO Project for their continued funding and support. The TAIL IO project is an international cooperative research project led by StatoilHydro and an R\&D consortium consisting of ABB, IBM, Aker Solutions, and SKF.

P. J. From and J. T. Gravdahl are with the Department of Engineering Cybernetics, Norwegian University of Science and Technology, 7491 Trondheim, Norway (e-mail: from@itk.ntnu.no).

J. Gunnar is with the Department of Strategic R\&D for Oil, Gas and Petrochemicals, Division of Process Automation, ABB, Oslo N-0603, Norway.

Color versions of one or more of the figures in this paper are available online at http://ieeexplore.ieee.org.

Digital Object Identifier 10.1109/TASE.2010.2089450 details of the proposed approach and the expressions to be implemented are found in [1].

In [2], the idea of introducing the paint quality as a constraint and minimize some additional cost function was presented. This opens for the possibility of allowing a small error in the orientation of the end effector in order to increase the velocity of the paint gun, reduce torques, and so on. In [3], the problem of friction force limit constraints was transformed into a problem of testing for positive definiteness of a certain matrix and in [4] the same ideas were used to convert the problem of orientation error constraints into a test of positive definiteness of a matrix. For different types of orientation errors, a suitable matrix was found and it was shown that positive definiteness of this matrix is equivalent to an orientation satisfying the given restrictions. By transforming the nonlinear orientation constraints into positive definiteness constraints imposed on certain matrices we transformed the problem of finding the optimal orientation into an optimization problem on the smooth manifold of linearly constrained positive definite matrices [1].

In [1], From and Gravdahl showed that by allowing an orientation error of $20^{\circ}$ we are able to: 1) reduce the torques required to follow a path by about $50 \%$ and 2) increase the speed at which the end effector can follow a trajectory with about $50 \%$. These results were found through simulations. It is important to confirm these results also through empirical studies in the lab. In this paper, we have implemented the same algorithms on a robot and measured the torques needed to follow a typical path for painting a flat and curved surface.

\section{PRoblem Statement}

There are two main factors that play important roles in obtaining uniform paint coating in automotive manufacturing. The first is to move the paint gun with constant velocity throughout the trajectory. This is in general an easy task in following straight lines but can be a challenge in turns where high accelerations are required. The second factor is the orientation of the paint gun with respect to the surface, which should be orthogonal.

It can be shown that the velocity of the paint gun is far more important than the orientation when it comes to uniform paint coating. A small error $\left(<20^{\circ}\right)$ in the paint gun orientation does not affect the quality of the coating to the same extent as changes in the velocity. Based on these observations, we represent the orientation not as one frame, but as a constrained continuous set of frames. The problem treated in [1] is then formulated as follows.

Given a maximum allowed orientation error of the paint gun and a trajectory on the surface that the paint gun is to follow with constant velocity and with a fixed distance from the paint gun to the surface. Then, the problem is to find the orientation of the paint gun at every point on the trajectory that allows it to follow the trajectory with the highest possible constant velocity.

We consider a standard industrial manipulator. The first three joints are referred to as the main axes, or the main joints. These are the strongest joints and also the ones that require the most torque. While the main axes are mainly used for positioning the paint gun, the last three joints, referred to as the wrist joints, determine the orientation of the paint gun. We fix the inertial reference frame to the base of the manipulator. We also attach a frame to the end effector of the manipulator, in our case the paint gun. This is attached so that the end-effector $z$ axis is aligned with the direction of the paint flow. This axis is referred to as the central axis.

We thus follow the standard approach for defining the reference frames of the manipulator but instead of specifying only one frame, we can define a continuous set of frames that lie close to the original frame by some metric. The algorithm is then free to choose any frame 


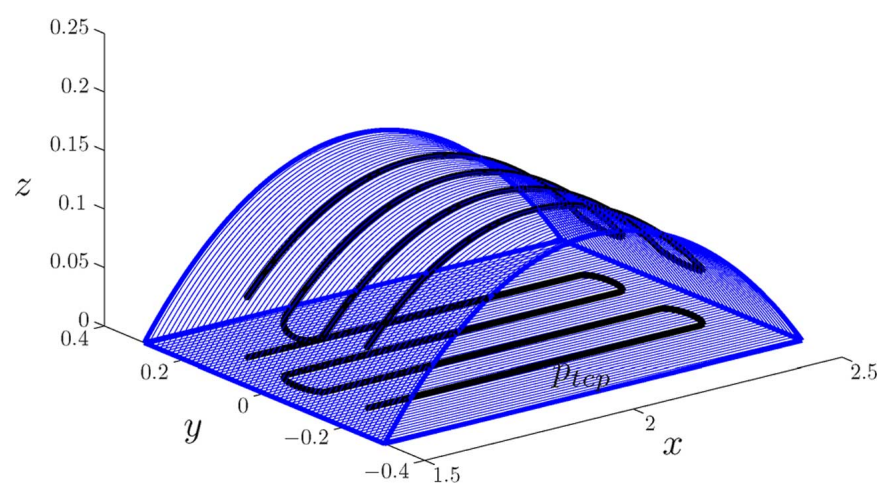

Fig. 1. The path of the tool center point (TCP) for the flat and the curved surface. Note the difference in scaling for the different axes.

that lies sufficiently close to the original frame. At each point in the path, the proposed algorithm then chooses the frame that results in the highest possible constant speed of the end effector.

For a spray paint robot following a path as the ones illustrated in Fig. 1, the main work load is on the main axes of the robot, i.e., joints 1,2 , and 3 . We observe that the work load on the wrist axes is very small compared to the main axes. The proposed algorithm will thus endeavour to move some of the work load from the main axes to the wrist axes. Because the joint torques of the main axes are very close to the torque limits, this should allow us to follow the trajectory with a higher velocity. Increasing the torques of the wrist axes should not pose any problems as these are very small for this type of trajectory.

\section{EXPERIMENTAL SET-UP}

All the experiments were performed in ABB's robot lab in Oslo, Norway on the ABB IRB-4400 industrial robot. The robot was equipped with an end effector of approximately the same weight as a spray paint gun. The optimal trajectories were computed offline to allow for analysis, but as far as the computation time is concerned, the computations could have been performed online. The resulting optimal trajectories were implemented in joint space feeding the joint positions for each joint at constant time intervals.

During the experiments, the positions, velocities, and torques of each joint were saved. All signals are scaled so that the maximum value equals 1 . The measurements for the three different approaches are scaled by the same factor, so the plots presented in the next section are comparable and illustrate well the difference between the different approaches.

The robot was set to follow a path which consists of four straight line segments and three turns. This path is defined by a series of points on the surface, denoted $p_{\text {tcp }}$. At every time $t$ the spray gun must point in the direction of $p_{\mathrm{tcp}}(t)$, i.e., at every time instance we specify a point at the surface which the central axis is to point in the direction of. This is the path of the tool center point (TCP). We perform the experiments for two different surfaces: 1) a flat surface in the $x y$-plane and 2) a curved surface. The surfaces are illustrated in Fig. 1. All the experiments are performed with a constant TCP velocity of $0.9 \mathrm{~m} / \mathrm{s}$. Note that the TCP velocity is the velocity of the trajectory at the surface and not of the point gun itself.

\section{EXPERIMENTAL RESULTS}

In this section, we present the experimental results when the trajectory is computed using the following algorithms.

1) Conventional - the orientation error is zero and the optimal orientation around the central axis is not utilized to improve performance.
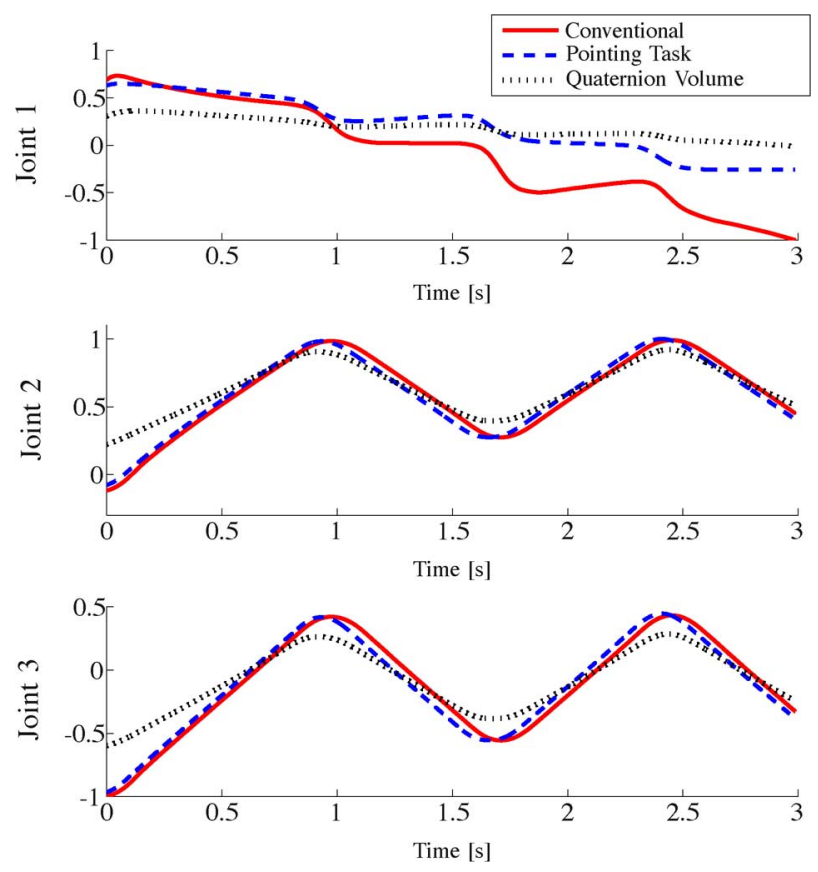

Fig. 2. Positions for the main axes. All positions are scaled.

2) Pointing Task - the orientation error is zero and the optimal orientation around the central axis is found. The rotation about the central axis can be chosen freely.

3) Quaternion Volume - an orientation error of $20^{\circ}$ is allowed and the optimal orientation is found. We also optimize around the central axis, as in 2).

\section{A. Flat Surface}

The joint positions for the main and wrist axes are found in Figs. 2 and 3 , respectively. We can see that the joint trajectories found by the three algorithms are quite different even though the end-effector position is basically the same and the orientation differs only slightly for the different approaches. The trajectories in joint space are quite similar for joints 2 and 3 , which is due to the kinematic coupling between these joints in the IRB-4400. The corresponding joint velocities are found in Figs. 4 and 5. Figs. 6 and 7 show the power for all the joints. We see clearly that the energy used is reduced for the main axes and that the wrist axes take more of the work load. The largest reduction is found in the first joint, which corresponds well with the position and velocity plots. We also note that for the wrist axes we use considerably more energy when an orientation error is allowed.

We use the square of the torque over the trajectory as a metric to compare the amount of torque needed to follow the three paths. This is given for each joint in Table I. We see that the square of the torques needed to follow the trajectory for the main axes decrease for the pointing task and even further for the quaternion volume. To show this more clearly, Table II shows the average of the square of the torques for the main and wrist axes. We see that for the main axes the square of the torques needed to follow the trajectory are reduced by $18 \%$ for the pointing task and $55 \%$ for the quaternion volume. This shows that the proposed algorithms work well and, as anticipated, the work load on the main axes is reduced substantially. For the wrist axes, however, the square of the torques increase. This is as expected, since the main objective was to move the work load from the main axes to the wrist axes. However, as the wrist torques needed to follow the trajectory for the conventional approach were so small, these are still well inside the torque limits and can still be considered very small, even though they increase by $117 \%$ 

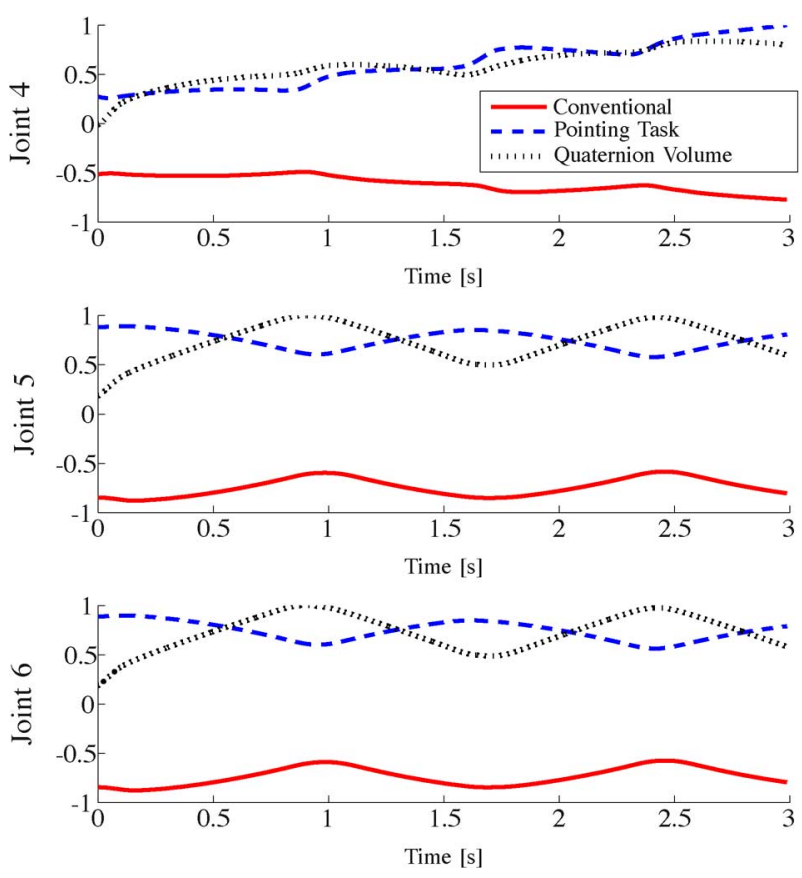

Fig. 3. Positions for the wrist axes. All positions are scaled.
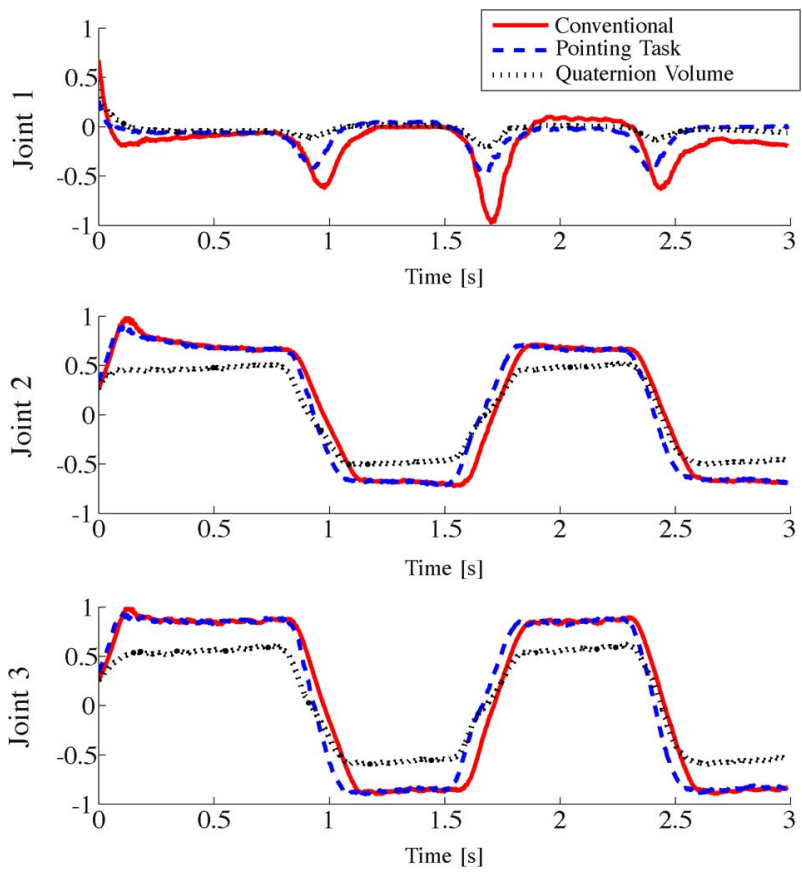

Fig. 4. Velocities for the main axes. All velocities are scaled.

and $83 \%$ for the pointing task and the quaternion volume, respectively. The average joint torque is shown in Table III.

The maximum and minimum torques are shown in Table IV. We see that for the main axes also the maximum values decrease for the pointing task and quaternion volume. As for the integral of the square of the torques, the maximum torques increase for the wrist axes. Again, these results are as expected and the work load is moved from the main axes to the wrist.

The maximum velocities for which we can follow the trajectory in Fig. 1 are shown in Table VII. We see that by optimizing the orientation around the central axis (the pointing task) at every time step, we
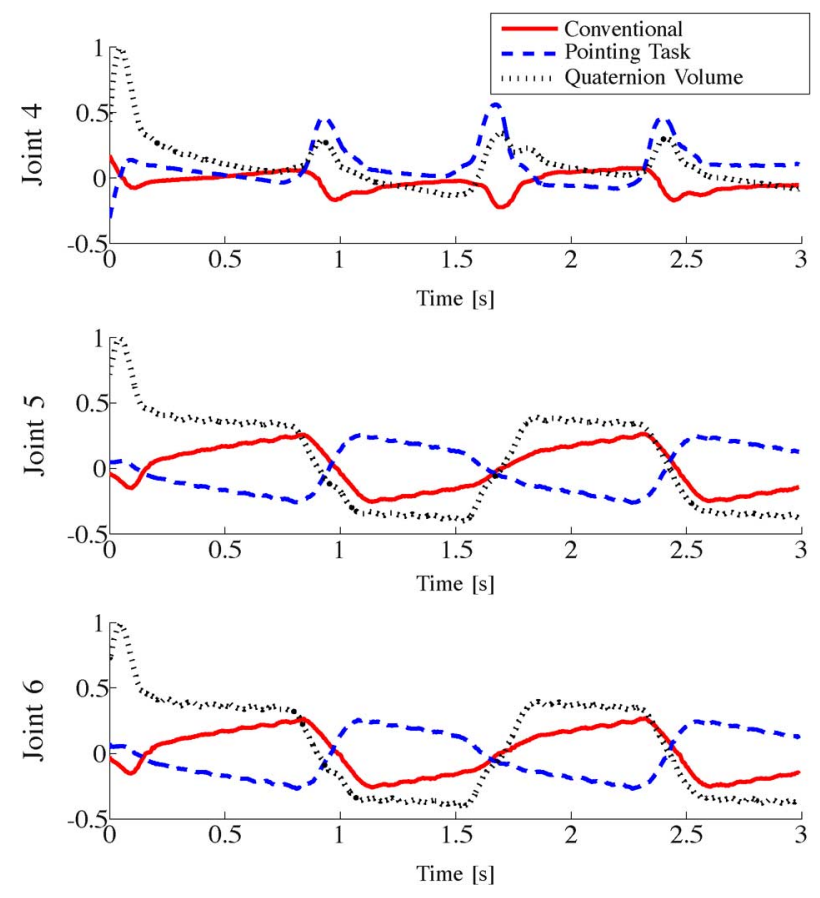

Fig. 5. Velocities for the wrist axes. All velocities are scaled.
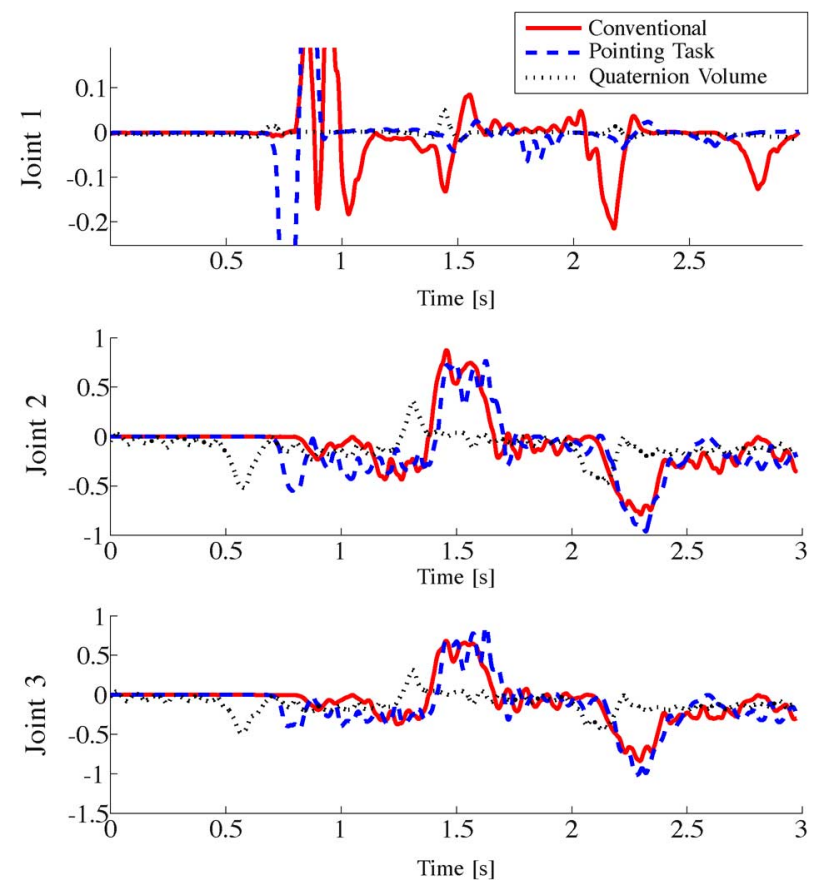

Fig. 6. The power of the main axes. All plots are scaled.

can increase the maximum speed slightly without allowing an orientation error. Any planning algorithm should thus include an optimization around the central axis to be able to increase the maximum speed or simply reduce the joint torques. However, if we allow an orientation error of about $20^{\circ}$ we see that we can increase the maximum speed with $50 \%$ compared to the conventional approach. Although the numbers are slightly different from the simulation results, the ratio between the numbers are approximately the same and the experimental results thus confirm the simulations presented by From and Gravdahl [1]. 

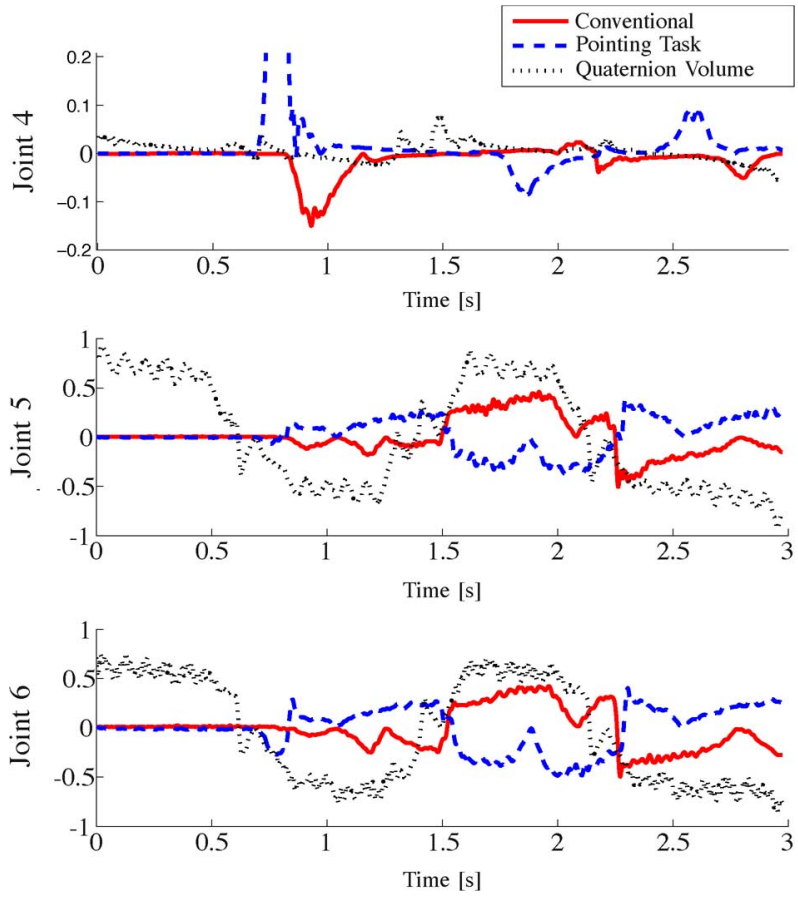

Fig. 7. The power of the wrist axes. All plots are scaled.

TABLE I

The SQuare of the Torques. All Values Are SCALED

\begin{tabular}{|c|c|c|c|}
\hline Joint & Conventional & Pointing Task & Quat Volume \\
\hline \hline 1 & 0.363 & 0.162 & 0.066 \\
\hline 2 & 1.000 & 0.881 & 0.518 \\
\hline 3 & 0.416 & 0.410 & 0.212 \\
\hline 4 & 0.010 & 0.012 & 0.021 \\
\hline 5 & 0.017 & 0.053 & 0.032 \\
\hline 6 & 0.011 & 0.014 & 0.011 \\
\hline
\end{tabular}

TABLE II

THE Average OF THE SQUARE OF THE TORQUES FOR THE MAIN AND WRIST AXES

\begin{tabular}{|c|c|c|c|}
\hline & Conventional & Pointing Task & Quat Volume \\
\hline \hline Main & 0.593 & $0.484(-18.4 \%)$ & $0.265(-55.3 \%)$ \\
\hline Wrist & 0.012 & $0.026(+116.6 \%)$ & $0.022(+83.3 \%)$ \\
\hline
\end{tabular}

TABLE III

THE AVERAge of THE SQUARE OF THE TORQUES FOR ALL THE JOINTS

\begin{tabular}{|c|c|c|c|}
\hline & Conventional & Pointing Task & Quat Volume \\
\hline All Joints & 0.303 & 0.255 & 0.143 \\
\hline
\end{tabular}

\section{B. Curved Surface}

The approach presented is not limited to planar surfaces. For curved surfaces such as the hood of a car, we can use the same approach. The path of the tool center point is a path on the curved surface and the direction of the end effector, assuming no orientation error, is set orthogonal to the surface at each point in the TCP path.

We see from Tables VIII and IX that for the curved surfaces we can reduce the square of the torque needed to follow the path even more than for flat surfaces. The square of the torques needed to follow a curved path are somewhat larger than for flat surfaces using the conventional approach, but at the same time the gain that we get from allowing
TABLE IV

THE MAXIMUM AND MINIMUM JOINT TORQUES OF ALL THE JOINTS FOR THE DifFERENT APPROACHES. All VALUES ARE NORMALIZED

\begin{tabular}{|c|c|c|c|c|c|c|}
\hline \multirow{2}{*}{ Joint } & \multicolumn{2}{|c|}{ Conventional } & \multicolumn{2}{c|}{ Pointing Task } & \multicolumn{2}{c|}{ Quat Volume } \\
\cline { 2 - 7 } & Max & Min & Max & Min & Max & Min \\
\hline \hline 1 & 1.000 & -0.483 & 0.752 & -0.355 & 0.555 & -0.211 \\
\hline 2 & 0.791 & -0.553 & 0.789 & -0.667 & 0.700 & -0.647 \\
\hline 3 & 0.452 & -0.486 & 0.520 & -0.592 & 0.329 & -0.495 \\
\hline 4 & 0.087 & -0.066 & 0.174 & -0.053 & 0.176 & -0.290 \\
\hline 5 & 0.065 & -0.069 & 0.433 & -0.100 & 0.191 & -0.160 \\
\hline 6 & 0.043 & -0.069 & 0.158 & -0.047 & 0.075 & -0.073 \\
\hline
\end{tabular}

TABLE V

The Average of the MaXimum AND Minimum of the MaIn AND Wrist AXES

\begin{tabular}{|c|c|c|c|c|c|c|}
\hline & \multicolumn{2}{|c|}{ Conventional } & \multicolumn{2}{c|}{ Pointing Task } & \multicolumn{2}{c|}{ Quat Volume } \\
\cline { 2 - 7 } & Max & Min & Max & Min & Max & Min \\
\hline \hline Main & 0.745 & -0.507 & 0.682 & -0.540 & 0.528 & -0.418 \\
\hline Wrist & 0.065 & -0.061 & 0.255 & -0.067 & 0.147 & -0.175 \\
\hline
\end{tabular}

TABLE VI

The Average of the MaXimum ANd Minimum TorQues

\begin{tabular}{|c|c|c|c|c|c|c|}
\hline & \multicolumn{2}{|c|}{ Conventional } & \multicolumn{2}{c|}{ Pointing Task } & \multicolumn{2}{c|}{ Quat Volume } \\
\cline { 2 - 7 } & Max & Min & Max & Min & Max & Min \\
\hline \hline All Joints & 0.406 & -0.284 & 0.471 & -0.302 & 1.337 & -0.296 \\
\hline
\end{tabular}

TABLE VII

The MaXimum SPeEd the Manipulator CAN Follow the Path AND the CORRESPONDING ORIENTATION ERRORS

\begin{tabular}{|l|c|c|}
\hline & Max vel $[\mathrm{m} / \mathrm{s}]$ & Max or. error $\left.{ }^{\circ}\right]$ \\
\hline Conventional & 0.94 & 0 \\
Pointing Task & 0.98 & 0 \\
Quaternion Volume & 1.41 & 20 \\
\hline
\end{tabular}

TABLE VIII

THE AVERAGE OF THE SQUARE OF THE TORQUES FOR THE MAIN JOINTS AND THE WRIST JOINTS WHEN APPLIED tO A CURVED SURFACE

\begin{tabular}{|c|c|c|c|}
\hline & Conventional & Pointing Task & Quat Volume \\
\hline \hline Main & 0.623 & $0.509(-18.3 \%)$ & $0.271(-56.5 \%)$ \\
\hline Wrist & 0.152 & $0.232(+52.6 \%)$ & $0.202(+32.9 \%)$ \\
\hline
\end{tabular}

TABLE IX

The Average of the MAXIMUM AND Minimum of the MaIN AND WRIST AXES WHEN APPLIED to A CURVED SURFACE

\begin{tabular}{|c|c|c|c|c|c|c|}
\hline & \multicolumn{2}{|c|}{ Conventional } & \multicolumn{2}{c|}{ Pointing Task } & \multicolumn{2}{c|}{ Quat Volume } \\
\cline { 2 - 7 } & Max & Min & Max & Min & Max & Min \\
\hline \hline Main & 0.904 & -0.677 & 0.792 & -0.702 & 0.596 & -0.520 \\
\hline Wrist & 0.277 & -0.223 & 0.288 & -0.167 & 0.224 & -0.201 \\
\hline
\end{tabular}

an orientation error is larger and the torques needed to follow the trajectory on a flat and curved surface are more or less the same when an orientation error of $20^{\circ}$ is allowed.

As expected the performance of the algorithm improves for a curved surface. This is mainly because for the curved surface the orientation of the paint gun changes over the path. Allowing a freedom in the specifications of the end-effector orientation allows us to "even out" these changes in the orientation and thus sweep over the surface more smoothly. 


\section{CONCLUSION}

Previous publications have suggested that a small error in the end-effector orientation does not decrease the quality of the paint job to a large extent. To guarantee uniform paint coating, it is far more important to maintain constant velocity throughout the trajectory. It is thus proposed to use the freedom that arises when we allow a small orientation error to increase the velocity of the end effector.

The preliminary work showed that one should be able to reduce the maximum torques and the energy needed to follow a specific path by about $50 \%$ by allowing a small orientation error in the specification of the end effector. The need to confirm these promising simulation results through experiments is thus apparent. In this paper, we have validated the theory and simulations presented previously and shown that we can substantially reduce the joint torques needed for a spray paint robot to follow a specific end-effector trajectory. We have shown that both the energy used and the maximum torques are reduced. This allows us to paint the surface considerably quicker than with the conventional approach.

In this paper, we have also investigated how the algorithm performs on curved surfaces. We are able to reduce the torques even more than for flat surfaces which shows that the approach is versatile and can be applied to a wide variety of problems.

\section{REFERENCES}

[1] P. J. From and J. T. Gravdahl, "A real-time algorithm to determine the optimal paint gun orientation in spray paint applications," IEEE Trans. Autom. Sci. Eng., vol. 7, no. 4, pp. 803-816, Oct. 2010, doi: 10.1109/TASE.2009.2033567.

[2] V. Potkonjak, G. Dordevic, D. Kostic, and M. Rasic, "Dynamics of anthropomorphic painting robot: Quality analysis and cost reduction," Robot. Autonomous Syst., vol. 32, no. 1, pp. 1493-1499, 2000.

[3] M. Buss, H. Hashimoto, and J. B. Moore, "Dextrous hand grasping force optimization," IEEE Trans. Robot. Autom., vol. 12, no. 3, pp. 406-418, 1996.

[4] P. J. From and J. T. Gravdahl, "On the equivalence of orientation error and positive definiteness of matrices," in Proc. Int. Conf. Control, Autom., Robot. Vision, Hanoi, Vietnam, 2008, pp. 2089-2094.

\section{A Framework to Model the Topological Structure of Supply Networks}

Qi Xuan, Fang Du, Yanjun Li, and Tie-Jun Wu, Member, IEEE

Abstract-Topological structure is considered more and more important in managing a supply network or predicting its development. In this paper, a new framework is proposed to model the topological structure of supply networks, where different types of supply networks can be created just by introducing different supplier-customer connecting rules. Generally, the networks created in the framework are much different from the random networks with the same degree sequences. The revealed phenomenon suggests that real-world supply networks may benefit from its intrinsic mechanism on flexibility, efficiency, and robustness to target attacks.

Note to Practitioners-The topological structure of supply networks is considered more and more important in managing a supply network or predicting its development. In this paper, we introduce a framework to model and analyze the topological structure of supply networks. This work aims to characterize supply networks by statistical methods and can help researchers better understand the material dynamics on supply networks and further conveniently create their own supply networks by summarizing practical supplier-customer connecting rules or analyzing real-world supply network data. The work should be further expanded in other aspects, such as simulating material dynamics on supply networks, designing optimal structure by introducing proper supplier-customer connecting rules, rearranging local connections to enhance the competitiveness and further ensure the long-term benefit of a target firm, and so on, all of which are of much interest for governors, investors, and managers and can be studied in the present framework in the future.

Index Terms-Complex network, logistics, modeling, self-organized system, supply network.

\section{INTRODUCTION}

$\mathbf{P}$ AST research on supply chains generally focused on optimizing the physical flow of materials [1]. However, nowadays, increasing product/service complexity, customer expectation, outsourcing and globalization lead to increasingly complex and dynamic supply networks. In such a situation, those exactly analytical approaches are losing their effects. Recently, several modern simulating methods, such as multiagent system (MAS) [2] and complex adaptive system (CAS) [3], [4] were introduced to model such increasingly complex supply networks. Provided with a set of proper rules, an artificial supply network can be dynamically created, then the coming state of real supply networks can be somewhat predicted and controlled by the model.

Manuscript received November 23, 2009; revised May 26, 2010; accepted August 15, 2010. Date of publication September 13, 2010; date of current version April 06, 2011. This paper was recommended for publication by Associate Editor C. Chu and Editor Y. Narahari upon evaluation of the reviewers' comments. This work was supported in part by the National Natural Science Foundation of China under Grant 61004097 and the China Postdoctoral Science Foundation under Grant 20080441256.

Q. Xuan is with the Department of Automation, Zhejiang University of Technology, Hangzhou 310023, China (e-mail: crestxq@hotmail.com).

F. Du and T.-J. Wu are with the Department of Control Science and Engineering, Zhejiang University, Hangzhou 310027, China (e-mail: fdu@iipc.zju. edu.cn; tjwu@zju.edu.cn).

Y. Li is with the School of Information and Electrical Engineering, Zhejiang University City College, Hangzhou 310015, China (e-mail: liyanjun @zucc.edu. cn).

Color versions of one or more of the figures in this paper are available online at http://ieeexplore.ieee.org.

Digital Object Identifier 10.1109/TASE.2010.2071414 